\title{
ALPHABET LORM - A CONSTRUÇÃO DE UM OBJETO DE APRENDIZAGEM PARA O APERFEIÇOAMENTO DE PROFESSORES NA COMUNICAÇÃO COM SURDOCEGOS
}

\author{
Márcia Debieux de Oliveira Lima e Lemes Soares ${ }^{1}$, Gunnar Correa ${ }^{2}$, Janiele de Souza Santos ${ }^{3}$, Danielle \\ Aparecida do Nascimento dos Santos ${ }^{4}$, Mario Augusto Pazoti ${ }^{5}$, Sônia Sanae Sato ${ }^{6}$ \\ ${ }^{1}$ Graduada em Psicologia pela Pontifícia Universidade Católica - PUC, São Paulo. Atua no Núcleo de Educação a Distância da Universidade do Oeste \\ Paulista- UNOESTE, Presidente Prudente, SP. E-mail: marcialemes@unoeste.br \\ ${ }^{2}$ Graduado em Sistemas Para Internet pela Universidade do Oeste Paulista - UNOESTE. Atua como Web Designer no Núcleo de Educação a \\ Distância da Universidade do Oeste Paulista- UNOESTE, Presidente Prudente, SP. \\ ${ }^{3}$ Graduada em Matemática pela Universidade Estadual Paulista - UNESP/Presidente Prudente. Mestranda pelo Programa de Pós-Graduação em \\ Educação da Universidade Estadual Paulista - UNESP/Presidente Prudente e atua junto ao Núcleo de Educação a Distância da Universidade do \\ Oeste Paulista- UNOESTE, Presidente Prudente, SP. \\ ${ }^{4}$ Doutora em Educação pela Universidade Estadual Paulista - UNESP/ Presidente Prudente. Professora do Programa de Pós-Graduação em \\ Educação da Universidade do Oeste Paulista - UNOESTE, Presidente Prudente, SP. \\ ${ }^{5}$ Mestre em Ciências da Computação e Matemática Computacional pela Universidade de São Paulo - USP. Professor da Faculdade de Informática \\ de Presidente Prudente (FIPP/UNOESTE), Presidente Prudente, SP. \\ ${ }^{6}$ Mestre em Engenharia de Produção pela Escola de Engenharia de São Carlos da Universidade de São Paulo - USP. Coordenadora Geral do Núcleo \\ de Educação a Distância - NEAD e de Cursos de Pós-Graduação Lato Sensu Presencial e EAD na Universidade do Oeste Paulista - UNOESTE, \\ Presidente Prudente, SP.
}

\section{RESUMO}

O aprimoramento da formação inicial e continuada de professores, tem se tornado cada dia mais imprescindível para garantir qualidade e eficiência aos processos inclusivos. Este aperfeiçoamento se torna ainda mais relevante quando pensamos em grupos cujas deficiências comprometem severamente partes motoras, cognitivas e/ou sensoriais, como a surdocegueira. Assim, o objetivo deste trabalho é apresentar a construção de um Objeto de Aprendizagem desenvolvido no Núcleo de Educação a Distância de uma Universidade do interior paulista, como recurso facilitador no processo de formação docente, para a aprendizagem dos fundamentos do Sistema Lorm, a fim de potencializar a comunicação com os estudantes surdocegos. Justifica-se a importância da utilização de recursos como o Alfhabet Lorm, construído com a intenção de facilitar o processo de formação docente em mais uma técnica comunicacional, possibilitando assim, a construção de modelos significativos para a inclusão de pessoas surdocegas, que muitas vezes se veem excluídas do processo educacional.

Palavras-chave: Objeto de Aprendizagem. Formação de Professores. Surdocego.

\section{ALPHABET LORM - THE CONSTRUCTION OF A LEARNING OBJECT FOR THE IMPROVEMENT OF TEACHERS IN THE COMMUNICATION WITH DEAF-BLIND}

\begin{abstract}
The improvement of the initial and continuous formation of teachers, has become more and more indispensable to guarantee quality and efficiency to the inclusive processes. This improvement becomes even more relevant when we think of groups whose impairments severely compromise motor, cognitive and/or sensory parts, such as deafblindness. Thus, the objective of this work is the construction of an object of Learning developed not at the Distance Learning Center of a University of the interior of the state of São Paulo, as a resource facilitator training process, for a learning of the funds of the Lorm System in order to Potentialize communication with the deafblind students. The use of resources such as the Lorm Alphabet, built with the intention of facilitating the process of teacher training in a more communicational technique, makes it possible to construct meaningful models for the inclusion of deafblind people, who often are excluded from the educational process.
\end{abstract}

Keyword: Learning Object. Teacher Training. Deafblind. 


\section{INTRODUÇÃO}

Garantir uma educação de qualidade para todos, tem sido uma tendência mundial que, a partir da década de 1990 vem se consolidando, e especialmente a partir da Conferência Mundial sobre Educação Inclusiva de 1994 - em Salamanca, na Espanha, vem determinando diretrizes básicas para a formulação e reforma de políticas, práticas e sistemas educacionais, com o foco na inclusão social e educacional.

Para responder a esse novo cenário educacional, o aprimoramento da formação inicial e a necessidade da formação continuada, tanto de professores da sala regular, como dos professores especialistas do Atendimento Educacional Especializado (AEE), vêm se mostrando imprescindíveis, para garantir que atendam, com respeito e eficiência, às especificidades da diversidade dos educandos.

Estes aprimoramentos se tornam ainda mais relevantes quando pensamos na inclusão social e educacional de Pessoas com Deficiência $(P D)$, especialmente, quando se trata de grupos cujas deficiências comprometem severamente partes motoras, cognitivas e/ou sensoriais, que dificultam ou mesmo impedem a comunicação, sobretudo quando a PD apresenta cognição preservada, como ocorre em alguns graus de paralisia cerebral, múltipla deficiência sensorial e surdocegueira.

Dessa forma, a elaboração, desenvolvimento e aprimoramento de estratégias de intervenção, devem fazer parte do cotidiano desses profissionais, visto que sua atuação deve, ainda, colaborar para que o surdocego desenvolva habilidades suficientes para a aquisição da linguagem.

Assim, quanto maior o leque de conhecimentos do professor, em diferentes métodos, técnicas e recursos, maiores serão as possibilidades de aprendizagem do surdocego, e o respeito por suas individualidades, ritmos e formas de aprendizagem.

Para tanto, no sentido de contribuir com a formação docente para o trabalho com estudantes surdocegos, foi proposta a elaboração de um Objeto de Aprendizagem (OA) - Alphabet Lorm para o curso de Pedagogia em Educação a Distância (EaD) de uma Universidade do interior paulista.

Como afirma Spinelli (2005),

Um objeto virtual de aprendizagem é um recurso digital reutilizável

\begin{abstract}
que auxilie na aprendizagem de algum conceito e, ao mesmo tempo, estimule o desenvolvimento de capacidades pessoais, como, por exemplo, imaginação e criatividade. Dessa forma, um objeto virtual de aprendizagem pode tanto contemplar um único conceito quanto englobar todo o corpo de uma teoria. Pode ainda compor um percurso didático, envolvendo um conjunto de atividades, focalizando apenas determinado aspecto do conteúdo envolvido, ou formando, com exclusividade, a metodologia adotada para determinado trabalho. (SPINELLI, 2005, p.7).
\end{abstract}

Portanto, o objetivo deste trabalho é apresentar a construção de um OA, como recurso facilitador no processo de formação docente, para a aprendizagem dos fundamentos do Sistema Lorm de Comunicação, a fim de potencializar a comunicação com os estudantes surdocegos.

Este artigo é resultado do PEIC (Protocolo: 3348/Data de submissão: 06/05/2016), vinculado ao Projeto de Pesquisa: Recursos Educacionais Digitais: perspectivas para uma escola inclusiva - graduação em Pedagogia EAD.

\subsection{Aspectos Específicos da Múltipla Deficiência Sensorial e da Surdocegueira}

Tanto a Múltipla Deficiência Sensorial quanto a Surdocegueira se caracterizam por apresentar limitações dos sentidos à distância, ou seja, perda parcial ou total da visão e audição. Segundo a Cartilha do Ministério da Educação (BRASIL, 2006) - Dificuldades de Comunicação e Sinalização - Surdocegueira/múltipla deficiência sensorial - a múltipla deficiência sensorial se diferencia da surdocegueira, pois além dos déficits auditivos e visuais está associada a outros comprometimentos físicos, intelectuais e/ou emocionais.

$\mathrm{Na}$ Conferência Mundial sobre surdocegueira de 1985, definiu-se que: 
Uma pessoa é surdocega quando tem um grau de deficiência visual e auditiva grave que the ocasiona sérios problemas na comunicação e mobilidade. Uma pessoa surdocega necessita de ajudas específicas para superar essas dificuldades na vida diária e em atividades educativas, profissionais e comunitárias. Incluem-se neste grupo, não somente as pessoas que têm perda total destes sentidos, como também aquelas que possuem resíduos visuais e/ou auditivos, que devem ser estimulados para que a sua "incapacidade" seja a menor possível (KELLER apud ALMEIDA 2015, p. 25).

A surdocegueira pode apresentar diferentes graus e causas de comprometimento sensorial. Segundo Almeida (2015), a surdocegueira pode ser dividida e classificada de várias maneiras, entre elas: quanto ao grau de perda sensorial: cegueira e surdez total; cegueira e surdez parcial (podendo esta ser profunda, severa ou moderada); entre outros; quanto às causas: pré-natal, perinatal e pós-natal e quanto ao período em que se manifestou - nesse caso, considera-se o comprometimento linguístico: congênita e adquirida.

Assim, compreender as possibilidades de comunicação de cada um e encontrar as melhores opções de comunicação é fundamental, para que o professor, mediador ${ }^{1}$ ou guiaintérprete ${ }^{2}$, possibilite o acesso do surdocego às informações.

Importante destacar que, sobretudo nos casos de surdocegueira congênita, ou nas

\footnotetext{
1 Aqui, o temo mediador refere-se ao profissional ou pessoa responsável por auxiliar o desenvolvimento da linguagem e as relações sociais do surdocego. Vale ressaltar que este pode ser um membro da família ou especialista da área.

${ }^{2}$ Profissional previsto pela Resolução SE 8, de 29/01/2016, para atuação nas escolas da rede estadual de ensino, com habilitação na Língua Brasileira de Sinais que assegura o acesso dos estudantes com deficiência auditiva, surdos ou surdocegos aos conteúdos desenvolvidos em ambiente escolares. Destaca-se que o guiaintérprete pode ter formação habilitada no magistério, ou qualquer curso a nível superior.
}

adquiridas na tenra infância, o papel do mediador pode colaborar significativamente com 0 estabelecimento de vínculos comunicacionais entre a criança surdocega e a família, contribuindo assim com o desenvolvimento e fortalecimento de suas relações afetivas e sociais. Pois são essas interações que vão possibilitar os processos de diferenciação do "eu" e do "outro" e a compreensão de seu mundo proximal, que neste caso, depende daquilo que o mediador ou a família conseguem apresentar de forma concreta. Apenas a partir desse processo de reconhecimento e construção de sua própria identidade e da capacidade de se comunicar com o mundo a sua volta, que se fundamentará a base para o desenvolvimento da linguagem - elemento primordial para todos os outros processos educativos e sociais.

Basicamente, os sistemas de comunicação dos surdocegos se dividem entre os que utilizam códigos orais, chamados de Sistemas não Alfabéticos, e aqueles que utilizam os códigos escritos - chamados de Sistemas Alfabéticos.

Segundo Dorado (2004) entre os Sistemas não alfabéticos encontram-se aqueles em que as palavras são representadas por sinais correspondentes, como por exemplo: Língua de Sinais Tátil (no caso do Brasil a Libras Tátil): o Método Tadoma; Língua de Sinais em campo reduzido (para aqueles que apresentam visão residual). $\mathrm{E}$ entre os Sistemas Alfabéticos podemos citar: o Alfabeto Datilológico Tátil - que pode ser utilizado em conjunto com a língua de sinais do respectivo país (no caso do Brasil a Libras Tátil); Escrita na palma das mãos (pode ser substituída pelo antebraço, ou mesmo pelas costas); e o Sistema Lorm e sua derivação Italiana que é o Método Malossi.

\subsection{Método Lorm}

O Método Lorm de Comunicação para surdocegos foi criado por volta de 1881, pelo escritor austríaco, Hieronymos Lorm (1821/1902) que perdeu a audição devido a uma grave doença aos 15 anos de idade e posteriormente, já por volta dos 40 anos, perdeu a visão. Para poder se comunicar, Lorm estabeleceu pontos de pressão na palma da mão e nos dedos, correspondentes às letras do alfabeto. Entretanto, o método que até hoje é muito utilizado nos países da Europa, sobretudo nos de língua alemã, só foi publicado por sua filha após sua morte.

Como mostra a Figura 1, o sistema utiliza 
a mão como tábua de digitação. Dessa forma, enquanto a pessoa que passa a informação vai apertando ou deslizando os dedos nos respectivos pontos, para formar as palavras, a pessoa que recebe a informação, fica com a palma da mão voltada para cima.

Da mesma forma, existem algumas particularidades do método que não necessitam da digitação letra a letra, como por exemplo, para dizer SIM e NÃO. Para o SIM, a pessoa que está "digitando" deve deslizar a mão aberta sobre a mão de quem está recebendo a informação, desde o seu pulso até as pontas dos dedos. E para o NÃO, a dinâmica é mesma, mas no sentido inverso - dos dedos para o pulso.

Como esse método é alfabético, seu aprendizado se torna mais fácil para pessoas que apresentam surdocegueira adquirida, principalmente para aqueles que foram alfabetizados, já para os surdocegos congênitos o aprendizado demanda uma maior atenção.

Figura 1. Pontos de cada letra do Alfabeto Lorm na palma da mão

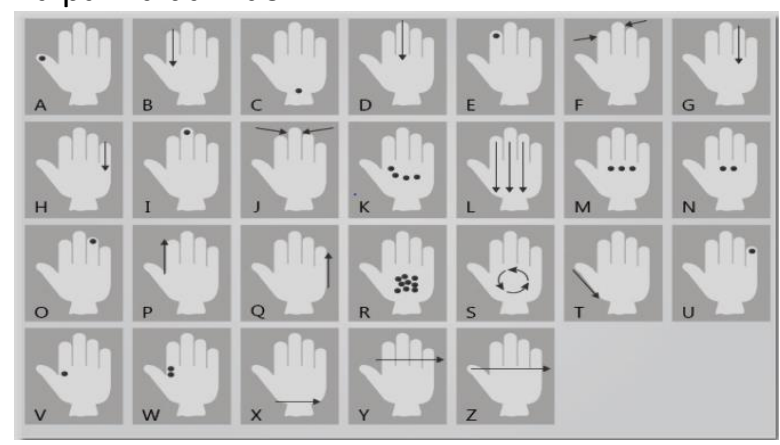

Fonte: NEAD (2017)

Para aprender o método, entretanto, o surdocego depende de um mediador que domine a técnica. Assim, pensando justamente na formação e aprimoramento de profissionais que podem cumprir esse papel, e aproveitando mais uma vez dos benefícios e oportunidades oferecidos pelas tecnologias, foi construído o Objeto de Aprendizagem Alphabet Lorm.

\section{METODOLOGIA}

Como já mencionado, o intuito deste trabalho é contribuir com a formação docente para o trabalho com estudantes surdocegos, a partir da utilização de recursos que facilitem a aprendizagem. Nesta perspectiva, como aporte teórico, buscamos auxílio de estudos e pesquisas da área da Surdocegueira, Formação de Professores e Educação Especial e Inclusiva. Assim, desenvolvemos a construção de um OA, descrito a seguir.

\subsection{Construção do Objeto de Aprendizagem - Alphabet Lorm}

Para o desenvolvimento do OA Alphabet Lorm foi utilizado o software Scirra Construct 2, Esse software permite desenvolver games, jogos e OA sem a necessidade de escrever linhas de códigos, deixando como responsabilidade para o programador pensar apenas na lógica e mecânica do objeto.

O jogo apresenta sinais gráficos - pontos e diferentes tipos de setas-, que quando selecionados, simulam em uma luva virtual, os movimentos e toques utilizados pelo Sistema Lorm de Comunicação.

Os pontos e setas (sinais gráficos) seguem, no plano cartesiano, a posição em $X$ e $Y$ do cursor do mouse, ou seja, conforme o usuário posicionar o cursor na luva digital, o ponto ou seta anteriormente selecionado, aparecerá sobre a luva. Mas, apenas quando o jogador escolher $\mathrm{e}$ clicar em determinada região da luva, O OA validará o tipo de movimento que se quer simular, fazendo assim, a correspondência e inserido no campo de texto a letra representada pelo toque ou movimento especificado.

Ao iniciar o jogo, instruções de utilização são apresentadas, bem como as letras do alfabeto e seus respectivos sinais gráficos dispostos na luva virtual, como pode se observar na Figura 2.

Durante o seu desenvolvimento foram realizados testes para garantir que sua navegabilidade e funcionalidade pudessem ser compreendidas mesmo por uma pessoa leiga, que nunca tivesse ouvido falar no Método Lorm, como mostra a Figura 3.

Figura 2. Sinais gráficos (setas e pontos)

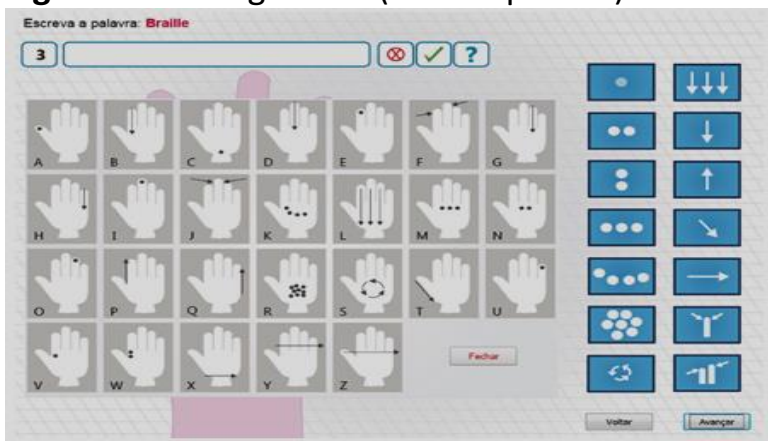

Fonte: NEAD (2017 
Figura 3. Interface Gráfica do AO

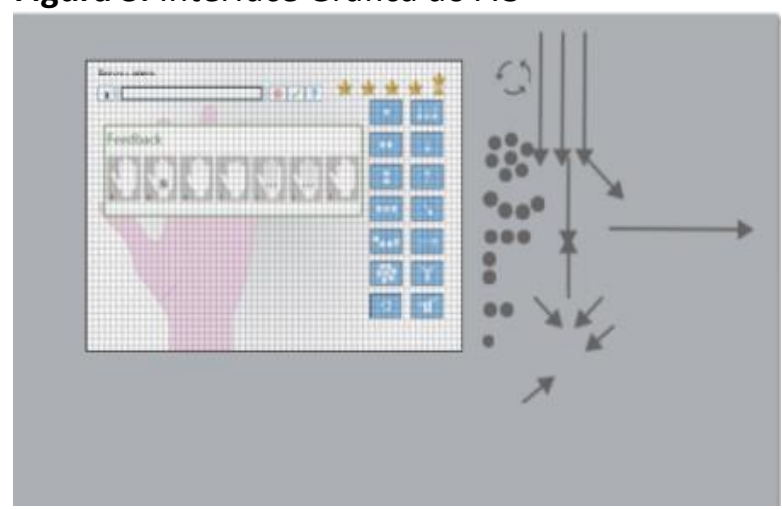

Fonte: NEAD (2017)

\section{RESULTADOS}

Após alguns meses de trabalho foi possível finalizar a interface gráfica do $\mathrm{OA}$, como mostra a Figura 4, bem como toda a representação do alfabeto Lorm e seus pontos na luva virtual.

Figura 4. Alfabeto Lorm na luva virtual

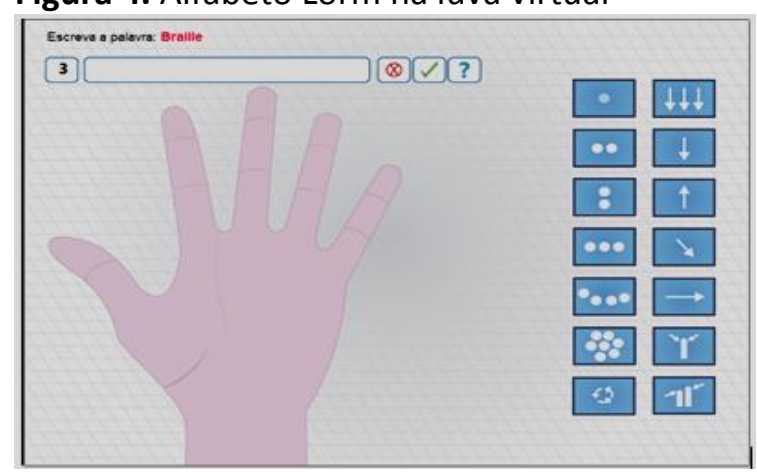

Fonte: NEAD (2017)

As Figuras 5 e 6 mostram que, para cada letra, um ponto, seta ou determinada região da luva, deverão ser escolhidos. Quando o usuário preencher com as respectivas letras todos os campos da palavra sugerida, o OA valida e verifica se as letras e, consequentemente, a palavra formada estão corretas.

Figura 5. Letra B da palavra Braille

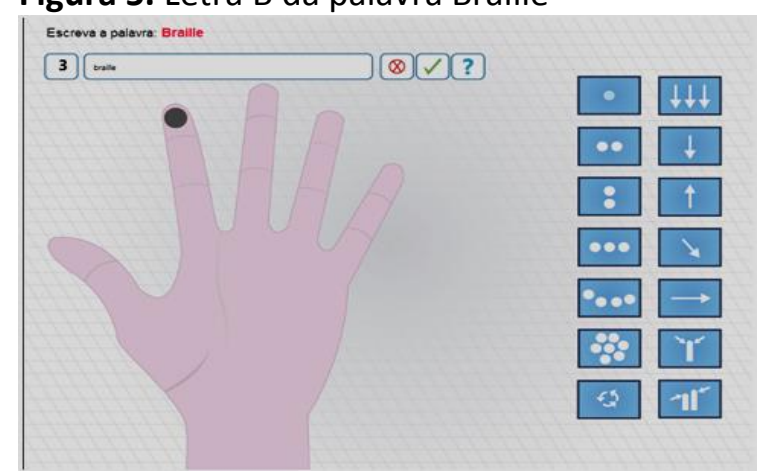

Fonte: NEAD (2017)
Figura 6. Letra E da palavra Braille

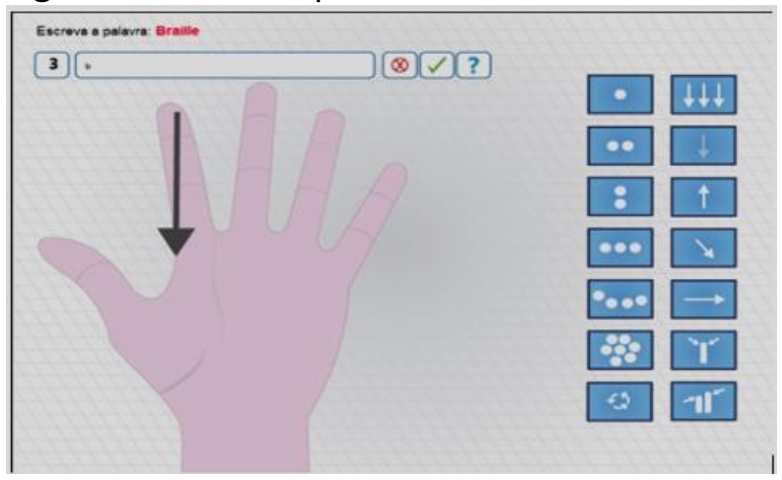

Fonte: NEAD (2017)

Se a palavra estiver correta, uma nova palavra é apresentada, mas se houver algum erro, são oferecidas três chances de correção de letras para cada palavra. Desse modo, assim que o usuário finalizar todas as palavras sugeridas pelo OA é apresentado o resultado final.

\section{DISCUSSÃO}

Considerando que o papel do professor pode colaborar significativamente com o desenvolvimento e fortalecimento dos processos comunicativos de pessoas com surdocegueira elemento primordial para todos os outros processos educativos e sociais - o desenvolvimento de técnicas e recursos que ampliem seu leque de conhecimentos e habilidades são de extrema importância.

Portanto, a necessidade desse acompanhamento personalizado exige uma formação específica e ao mesmo tempo ampla do professor, do mediador, bem como do guiaintérprete, de acordo com as premissas da Lei de Diretrizes e Bases da Educação Nacional (LDBEN/96), visto que para responder com qualidade a esse novo modelo educativo, é responsabilidade da escola se adequar e responder às necessidades de todos os estudantes (BRASIL, 1996).

Assim, embora o professor da sala comum não tenha que ser especialista - como os professores do AEE e guias-intérpretes - os processos formativos iniciais dos profissionais da educação básica já vem apresentando uma preocupação e oferecendo em sua grade curricular disciplinas específicas - como Braille, Libras, Tecnologia Assistiva, entre outras -, na perspectiva de ampliar tanto suas reflexões e saberes docentes, como instrumentalizá-los para a intervenção pedagógica, em função de um público-alvo cada vez mais heterogêneo. 


\section{CONCLUSÃO}

Nos espaços escolares a utilização de recursos que auxiliem o desenvolvimento dos processos de ensino e aprendizagem se torna imprescindível, e a partir das especificidades e possibilidades de cada caso, novas metodologias e novas práticas de ensino devem ser estabelecidas. Por isso, pensando no trabalho de pessoas surdocegas é primordial que professores, mediadores e guias-intérpretes tenham um profundo conhecimento de aspectos linguísticos como símbolos, signos e seus significados, bem como sobre as diferentes técnicas e métodos de comunicação.

Desse modo, justifica-se a importância da utilização de recursos como o OA Alfhabet Lorm, construídos com a intenção de facilitar o processo de formação docente em mais uma técnica comunicacional, possibilitando assim, a construção de modelos significativos para a inclusão de pessoas com surdocegueira que, muitas vezes, ainda se veem excluídas do processo educacional.

\section{REFERÊNCIAS}

ALMEIDA, W.G. O Guia-intérprete e a Inclusão da pessoa com surdocegueira. 2015. 187f. Tese (Doutorado em Educação) - Universidade Federal da Bahia, Salvador, 2015.

BRASIL. Ministério da Educação. Dificuldades de comunicação e sinalização: surdocegueira e múltipla deficiência sensorial. Brasília: MEC, 2006. (Coleção saberes e práticas de inclusão).

BRASIL. Lei Federal no 9.394/96 - Diretrizes e Bases da Educação Nacional. Brasília, 1996. Disponível em http://www.planalto.gov.br/ccivil 03/leis/L9394. htm. Acesso em: 25 jun 2017.

BRASIL. Resolução SE 8, de 29/01/2016. Dispõe sobre a atuação de docentes com habilitação/qualificação na Língua Brasileira de Sinais. Brasília, 2016. Disponível em http://siau.edunet.sp.gov.br/ItemLise/arquivos/8 16. HTM?Time $=12 / 05 / 2017 \% 2008: 28: 54$. Acesso em: 25 jun 2017.

DORADO, G.M. Sistemas de comunicación de personas sordociegas. In: REYES D.A. La Sordocegueira: um análisis multidisciplinar. Madrid: ONCE, 2004. P.193-252.
SPINELLI, W. Aprendizagem matemática em contextos significativos: objetos virtuais de aprendizagem e percursos temáticos. 2005. 138f. Dissertação (Mestrado em Educação) Universidade de São Paulo, São Paulo, 2005.

Recebido para publicação em: 03/10/2017

Revisado em: 06/11/2017

Aceito em: 13/11/2017 